\title{
Rayleigh Imaging of Graphene and Graphene Layers
}

\section{Casiraghi, ${ }^{\dagger}$ A. Hartschuh, ${ }^{\ddagger}$ E. Lidorikis,,$\stackrel{\$}{ }$ H. Qian, ${ }^{\ddagger}$ H. Harutyunyan, ${ }^{\ddagger}$ T. Gokus, ${ }^{\ddagger}$ K. S. Novoselov," and A. C. Ferrari ${ }^{\star, t}$}

Cambridge University, Engineering Department, Cambridge, United Kingdom,

Chemistry and Biochemistry Department and CeNS, Ludwig-Maximilians-

University of Munich, Germany, Department of Materials Science and

Engineering, University of Ioannina, Greece, and School of Physics and Astronomy,

University of Manchester, Manchester, M13 9PL, United Kingdom

Received May 17, 2007; Revised Manuscript Received August 5, 2007

\begin{abstract}
We investigate graphene and graphene layers on different substrates by monochromatic and white-light confocal Rayleigh scattering microscopy. The image contrast depends sensitively on the dielectric properties of the sample as well as the substrate geometry and can be described quantitatively using the complex refractive index of bulk graphite. For a few layers $(<6)$, the monochromatic contrast increases linearly with thickness. The data can be adequately understood by considering the samples behaving as a superposition of single sheets that act as independent two-dimensional electron gases. Thus, Rayleigh imaging is a general, simple, and quick tool to identify graphene layers, which is readily combined with Raman scattering, that provides structural identification.
\end{abstract}

Graphene is the prototype two-dimensional carbon system. ${ }^{1}$ Its electron transport is described by the (relativistic-like) Dirac equation, and this allows access to the rich and subtle physics of quantum electrodynamics in a relatively simple condensed matter experiment. ${ }^{2-6}$ The scalability of graphene devices to true nanometer dimensions ${ }^{7-9}$ makes it a promising candidate for future electronics because of its ballistic transport at room temperature combined with chemical and mechanical stability. Remarkable properties extend to bilayers and few layers. ${ }^{5,6,10-12}$ More fundamentally, the various forms of graphite, nanotubes, and buckyballs can all be viewed as derivatives of graphene.

Graphene samples can be obtained from micromechanical cleavage of graphite. ${ }^{2}$ Alternative procedures include chemical exfoliation of graphite ${ }^{13-17}$ or epitaxial growth by thermal decomposition of $\mathrm{SiC} .^{11,18-20}$ The latter has the potential of producing large-area lithography compatible films but is substrate limited. It is hoped that, in the near future, efficient large-area, substrate-independent growth methods will be developed, as is now the case for nanotubes.

Despite the wide use of micromechanical cleavage, the identification and counting of graphene layers is still a major hurdle. Monolayers are a minority among accompanying

\footnotetext{
* Corresponding author. E-mail: acf26@eng.cam.ac.uk.

Cambridge University, Engineering Department.

$\doteqdot$ Chemistry and Biochemistry Department and CeNS, Ludwig-Maximilians- University of Munich.

$\S$ Department of Materials Science and Engineering, University of Ioannina.

"School of Physics and Astronomy, University of Manchester.
}

thicker flakes. ${ }^{1}$ They cannot be seen in an optical microscope on most substrates. Currently, optically visible graphene layers are obtained by placing them on the top of oxidized $\mathrm{Si}$ substrates with typically $300 \mathrm{~nm} \mathrm{SiO}_{2}$. atomic force microscopy (AFM) is viable but has a very low throughput. Moreover, the different interaction forces between the AFM probe, graphene, and the $\mathrm{SiO}_{2}$ substrate lead to an apparent thickness of $0.5-1 \mathrm{~nm}$ even for a single layer, ${ }^{2,5}$ much bigger that what is expected from the interlayer graphite spacing. Thus, in practice, it is only possible to distinguish between one and two layers by AFM if graphene films contain folds or wrinkles. ${ }^{2,5}$ High-resolution transmission electron microscopy is the most direct identification tool, ${ }^{21,22}$ however, it is destructive and very time-consuming, being viable only for fundamental studies. ${ }^{21}$

Optical detection relying on light scattering is especially attractive because it can be fast, sensitive, and not destructive. Light interaction with matter can be elastic or inelastic, and this corresponds to Rayleigh and Raman scattering, respectively. Raman scattering has recently emerged as a viable, nondestructive technique for the identification of graphene and its doping. ${ }^{22,23}$ However, Raman-scattered photons are a minority compared to those elastically scattered. Here, we show that the elastically scattered photons provide another very efficient and quick means to identify single and multilayer samples and a direct probe of their dielectric constant.

Rayleigh scattering was previously used to monitor size, shape, concentration, and optical properties of nanoparticles, 


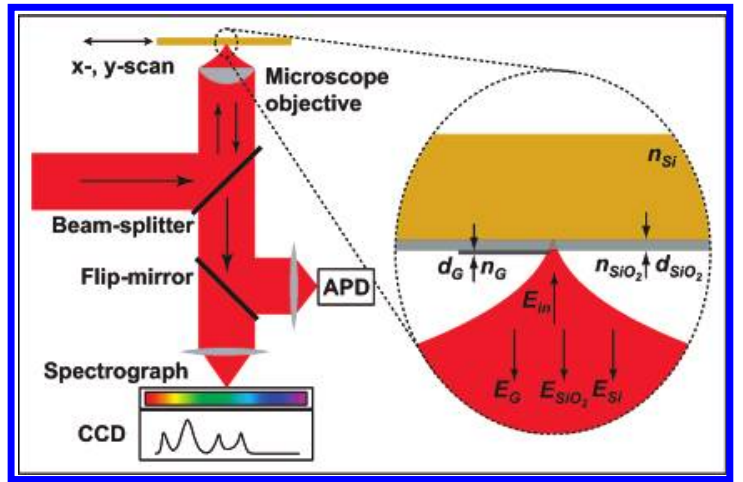

Figure 1. Schematic experimental setup for combined Rayleigh and Raman spectroscopy. The inset shows a cross-sectional view of the interaction between the optical field and graphene deposited on $\mathrm{Si}$ covered with $\mathrm{SiO}_{2}$.

carbon nanotubes, and viruses. ${ }^{24-28}$ Rayleigh scattering experiments can be performed by using two different strategies. In one, the background signal is minimized by making free-standing samples, as done in the case of carbon nanotubes, ${ }^{27,28}$ or by dark-field configurations. ${ }^{29}$ Alternatively, the background intensity is utilized as a reference beam, while the sample signal is detected interferometrically. ${ }^{24-26,30,31}$ Here, we combine the second approach with the interferometric modulation of the contributing fields and we show that the presence of a background is essential to enhance the detection of graphene over a certain wavelength range.

Graphene samples are produced by micromechanical cleavage of bulk graphite and deposited on a Si substrate covered with $300 \mathrm{~nm} \mathrm{SiO} 2$ (IDB Technologies Ltd). The sample thickness is independently confirmed by a combination of AFM and Raman spectroscopy. AFM is performed in tapping mode under ambient conditions. Raman spectra are measured at $514 \mathrm{~nm}$ using a Renishaw micro-Raman 1000 spectrometer. Rayleigh scattering is performed with an inverted confocal microscope, Figure 1. Either a $\mathrm{He}-\mathrm{Ne}$ laser $(633 \mathrm{~nm})$ or a collimated white-light beam are used as the excitation source. Coherent white-light pulses are generated by pumping a photonic crystal fiber with the output of a Ti:Sa oscillator operating at $760 \mathrm{~nm}$. The beam is reflected by a beam splitter and focused by a microscope objective with high numerical aperture $(\mathrm{NA}=0.95)$. However, the objective lens is not totally filled, which results in an effective NA $\sim 0.7$, thereby increasing the image contrast as discussed at the end of this paper. The scattered light from the sample is collected in backscattering geometry, transmitted by a beam splitter and detected by a photon-counting avalanche photodiode (APD), Figure 1. Alternatively, the reflected light is filtered using a notch filter to remove the laser excitation and sent to a spectrometer. This allows simultaneous Rayleigh and Raman measurements, Figures 1, 2a. Confocal Rayleigh images are obtained by raster scanning the sample with a piezoelectric scan stage. The acquisition time per pixel varies from a few milliseconds in the case of Rayleigh scattering to few minutes for Raman scattering. This empirically indicates that Rayleigh measurements are almost 5 orders

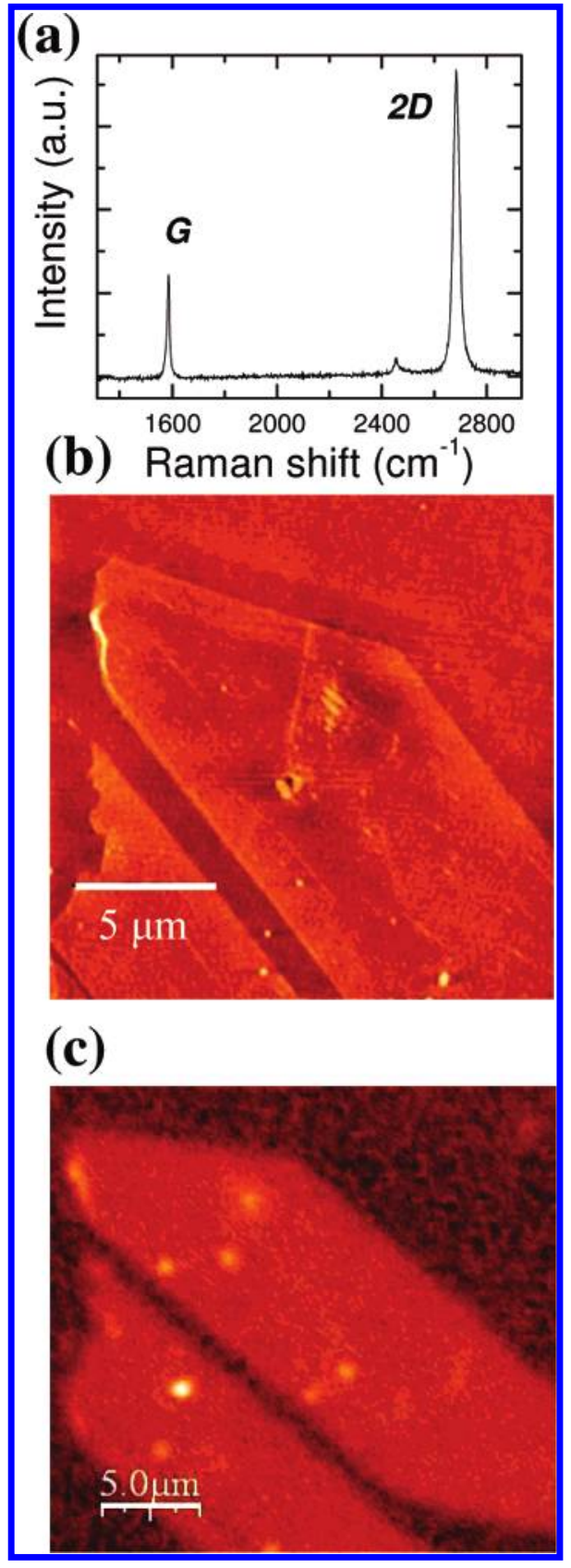

Figure 2. (a) Raman spectrum at $514 \mathrm{~nm}$, showing the features of graphene. $^{22}$ (b) AFM image of single layer graphene. (c) Confocal Rayleigh image obtained by raster scanning the sample with a piezoelectric scan stage. Note: The contrast of the Rayleigh image is reversed for easier comparison with the AFM image

of magnitude quicker than Raman measurements. The spatial resolution is $\sim 800 \mathrm{~nm}$.

Figure $2 \mathrm{~b}$ shows an AFM image of monolayer graphene. The AFM cross section gives an apparent height of $\sim 0.6 \mathrm{~nm}$. Raman spectroscopy confirms that the sample is a single layer (Figure 2a). ${ }^{22}$ Figure $2 b$ is the corresponding confocal Rayleigh image obtained with monochromatic laser light $(633 \mathrm{~nm})$. Figure $3 \mathrm{a}$ shows an optical micrograph of a sample composed of a varying number of layers. Once the single layer is identified by Raman scattering, we get the total number of layers from the measured AFM height by considering the interlayer spacing of $\sim 0.33 \mathrm{~nm}: z(\mathrm{~nm})=$ 


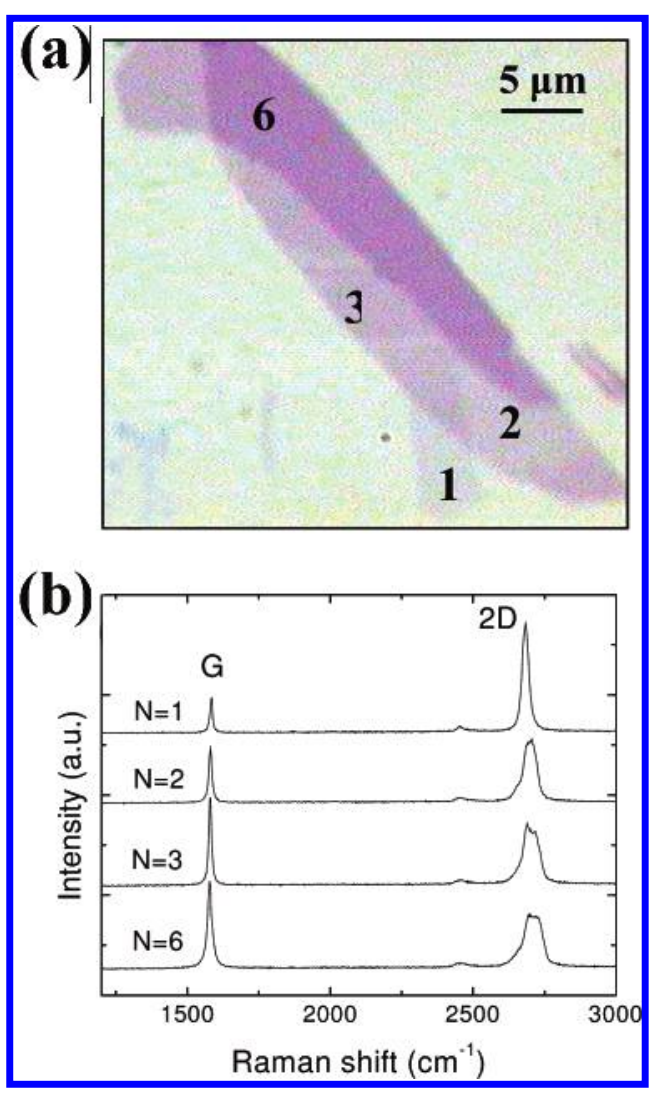

Figure 3. (a) Optical micrograph of multilayer with 1, 2, 3, and 6 layers. (b) Raman spectra as a function of number of layers.

$0.27+0.33 N$. This confirms that the sample is composed of 1,2, 3, and 6 layers, as for Figure 3a. These layers have a slightly different color in the optical microscope (Figure 3a). It appears that the darker color corresponds to the thicker sample. Note, however, that the color of much thicker layers (more than 10 layers) does not follow this trend and can change from blue to yellow to gray. The number of layers is further confirmed by the evolution of the $514 \mathrm{~nm}$ Raman spectra, ${ }^{22}$ Figure $3 b$. Figure $4 a$ shows a confocal Rayleigh map for $633 \mathrm{~nm}$ excitation. The signal intensity of in Figure 4 appears to increase with $N$.

We now discuss the physical origin of the image contrast $(\delta)$. This is defined as the difference between substrate and sample intensity, normalized to the substrate intensity. The single-layer contrast at $633 \mathrm{~nm}$ is $\sim 0.08$. The contrast is positive, i.e., the detected intensity from graphene is smaller than that of the substrate. The Rayleigh images in Figures $2 \mathrm{c}$ and $4 \mathrm{a}$ are reversed for convenience in order to compare them with AFM.

We explain the sign and scaling of the contrast for increasing $N$ in terms of interference from multiple reflections. The inset in Figure 1 shows a schematic of the interaction between the light and graphene on $\mathrm{Si}+\mathrm{SiO}_{2}$. When the light impinges on a multilayer, multiple reflections take place. ${ }^{32}$ Thus, the detected signal (I) results from the superposition of the reflected field from the air-graphene $\left(E_{\mathrm{G}}\right)$, graphene- $\mathrm{SiO}_{2}\left(E_{\mathrm{Si}_{2}}\right)$, and $\mathrm{SiO}_{2}-\mathrm{Si}$ interfaces $\left(E_{\mathrm{Si}}\right)$. The background signal $\left(I_{\mathrm{Bg}}\right)$ results from the superposition

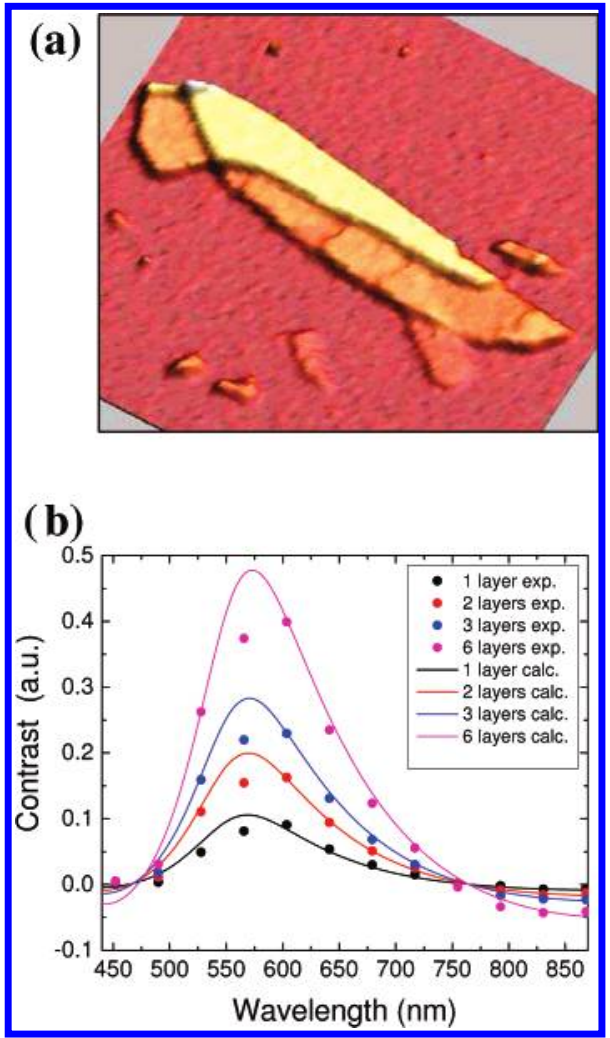

Figure 4. (a) Three-dimensional confocal Rayleigh map for monochromatic $633 \mathrm{~nm}$ excitation. The window size is $49 \mu \mathrm{m} \times$ $49 \mu \mathrm{m}$. (b) Experimental (dots) and theoretical (line) contrast as a function of excitation wavelength. Note: The contrast of the Rayleigh image data is reversed.

of the reflected field from the air- $\mathrm{SiO}_{2}$ interface and the Si substrate.

Before giving a complete quantitative model, it is useful to consider a simplified picture that captures the basic physics and illustrates why a single atomic layer can be visualized optically. The field at the detector is dominated by two contributions: the reflection by the graphene layer and the reflection from the $\mathrm{Si}$ after transmission through graphene and after passing through the $\mathrm{SiO}_{2}$ layer twice. Thus, the intensity at the detector can be approximated as:

$$
I \sim\left|E_{\mathrm{G}}+E_{\mathrm{Si}}\right|^{2}=\left|E_{\mathrm{G}}\right|^{2}+\left|E_{\mathrm{Si}}\right|^{2}+2\left|E_{\mathrm{G}}\right|\left|E_{\mathrm{Si}}\right| \cos \phi
$$

where $\phi$ is the total phase difference. This includes the phase change due to the optical path length of the oxide, $d_{\mathrm{SiO}_{2}}$, and that due to the reflection at each boundary, $\vartheta_{\mathrm{Si}}$ and $\vartheta_{\mathrm{G}}$ :

$$
\phi=\vartheta_{\mathrm{G}}-\left(\vartheta_{\mathrm{Si}}+2 \pi n_{\mathrm{SiO}_{2}} 2 d_{\mathrm{SiO}_{2}} / \lambda_{0}\right)
$$

where $n_{\mathrm{SiO}_{2}}$ is the refractive index of the oxide and $\lambda_{0}$ is the wavelength of the light in vacuum. Assuming the field reflected from graphene to be very small, $\left|E_{\mathrm{G}}\right|^{2} \simeq 0$, the image contrast $\delta$ results from interference with the strong field reflected by the silicon:

$$
\delta=\left(I_{\mathrm{Si}}-I\right) / I_{\mathrm{Si}} \simeq-2 \cdot\left|E_{\mathrm{G}}\right| /\left|E_{\mathrm{Si}}\right| \cdot \cos \phi
$$


The sign of $\delta$ depends on the sign of $\cos \phi$, which is given by eq 2 . The reflectance, $R$, is the ratio between the reflected power to the incident power. ${ }^{32}$ Assuming the Si reflectance as one, eq 3 can be written as:

$$
\delta=-2 \sqrt{ } R_{\mathrm{G}} \cos \phi
$$

where $R_{\mathrm{G}}$ is the reflectance of graphene. This is in turn related to the reflection coefficient $r_{\mathrm{G}}:{ }^{32}$

$$
r_{\mathrm{G}}=\sqrt{ } R_{\mathrm{G}} \cdot \exp \left(i \vartheta_{\mathrm{G}}\right)
$$

Equation 4 shows that the main role of the $\mathrm{SiO}_{2}$ is to act as a spacer: the contrast is defined by the phase variation of the light reflected by the $\mathrm{Si}^{33}$ Thus, the contrast for a given wavelength can be tailored by adjusting the spacer thickness or its refractive index.

To investigate the wavelength dependence of the image contrast, we perform Rayleigh spectroscopy with a whitelight source. A grating is used to analyze the detected light. Figure $4 \mathrm{~b}$ shows that, for $N=1$, the contrast is maximum at $\sim 570 \mathrm{~nm}$. The contrast at $633 \mathrm{~nm}$ is $\sim 0.08$, in agreement with the monochromatic Rayleigh scattering experiment. The contrast is zero at $750 \mathrm{~nm}$, and it is small and negative for $\lambda>750 \mathrm{~nm}$. From eqs 2 and 4 and assuming $\vartheta_{\mathrm{Si}}=-\pi$, the phase of graphene is $\vartheta_{\mathrm{G}} \simeq-\pi$, as expected for an ultrathin film. ${ }^{32}$ The contrast decreases in the near IR (for $d_{\mathrm{SiO}_{2}}=$ $300 \mathrm{~nm}$ ) because the wavelength becomes larger than twice the optical path length provided by the $\mathrm{SiO}_{2}$ spacer. Figure $4 \mathrm{~b}$ shows that, while the contrast increases for increasing $N$, the phase remains constant.

We now present a more accurate model, with no assumptions, which describes the light modulation by multiple reflections based on the recurrent matrix method for reflection and transmission of multilayered films. ${ }^{34} \mathrm{We}$ calculate the total electric and magnetic fields in the various layers, applying the boundary conditions at every interface. The fields at two adjacent boundaries are described by a characteristic matrix. This depends on the complex refractive index and the thickness of the film and the angle of the incident light. ${ }^{34}$ By computing the characteristic matrix of every layer and taking into account the numerical aperture of the objective and the filling factor, it is possible to find the reflection coefficient for an arbitrary configuration of spacer (2) and substrate (3) and for any number of graphene layers $(G)$. Assuming two counter-propagating waves, the standard boundary conditions for the reflection coefficient of a normally incident wave is:

$$
R=\left|\frac{M_{12}}{M_{22}}\right|^{2}
$$

where

$$
\begin{aligned}
M_{12}= & {\left[\cos \phi_{\mathrm{G}} \cos \phi_{2}\left(1-\frac{n_{\mathrm{Air}}}{n_{3}}\right)-\sin \phi_{\mathrm{G}} \sin \phi_{2}\left(\frac{n_{\mathrm{G}}}{n_{2}}-\frac{n_{\mathrm{Air}} n_{2}}{n_{\mathrm{G}} n_{3}}\right)\right] } \\
& -i\left[\cos \phi_{\mathrm{G}} \sin \phi_{2}\left(\frac{n_{2}}{n_{3}}-\frac{n_{\text {Air }}}{n_{2}}\right)-\sin \phi_{\mathrm{G}} \cos \phi_{2}\left(\frac{n_{\mathrm{G}}}{n_{3}}-\frac{n_{\mathrm{Air}}}{n_{\mathrm{G}}}\right)\right] \\
M_{22} & =\left[\cos \phi_{\mathrm{G}} \cos \phi_{2}\left(1+\frac{n_{\text {Air }}}{n_{3}}\right)-\sin \phi_{\mathrm{G}} \sin \phi_{2}\left(\frac{n_{\mathrm{G}}}{n_{2}}+\frac{n_{\text {Air }} n_{2}}{n_{\mathrm{G}} n_{3}}\right)\right] \\
& -i\left[\cos \phi_{\mathrm{G}} \sin \phi_{2}\left(\frac{n_{2}}{n_{3}}+\frac{n_{\text {Air }}}{n_{2}}\right)+\sin \phi_{\mathrm{G}} \cos \phi_{2}\left(\frac{n_{\mathrm{G}}}{n_{3}}+\frac{n_{\text {Air }}}{n_{\mathrm{G}}}\right)\right]
\end{aligned}
$$

with $\phi_{\mathrm{G}}=2 \pi n_{\mathrm{G}} d_{\mathrm{G}} / \lambda_{0}$ and $\phi_{2}=2 \pi n_{2} d_{2} / \lambda_{0}$. For incidence at an angle $\theta$, with s-polarization (transverse electric field), the same formula applies with the substitution $n_{i} \rightarrow n_{i} \cos \theta_{i}$, while for p-polarization, every ratio changes $n_{i} / n_{j} \rightarrow n_{i} \cos$ $\theta_{j} / n_{j} \cos \theta_{i}$. The phases change in both s- and p-polarizations to $\phi_{\mathrm{G}}=2 \pi n_{\mathrm{G}} d_{\mathrm{G}} \cos \theta_{\mathrm{G}} / \lambda_{0}$ and $\phi_{2}=2 \pi n_{2} d_{2} \cos \theta_{2} / \lambda_{0}$. The angle $\theta_{i}$ for every layer is obtained from Snell's law: $\theta_{i}=$ $\arcsin \left(\sin \theta_{0} / n_{i}\right)$. In case any of the layers is absorbing (as in graphene and $\mathrm{Si}$ ), we need use an effective index $n_{i}^{\prime}=$ $f\left(n_{i}, \theta_{0}\right)$, which depends on the incident angle from vacuum $\theta_{0}{ }^{32,35}$ In this case, the corresponding refraction angle is $\theta_{i}$ $=\arcsin \left[\sin \theta_{0} / \operatorname{Re}\left(n_{i}^{\prime}\right)\right]$.

The matrix method requires as input the complex refractive index of the sample. The frequency dependent $\mathrm{Si}$ and $\mathrm{SiO}_{2}$ indexes are taken from ref 36 . For graphene, few layers graphene, and graphite, this is anisotropic depending on the polarization of the incident light. For the electric field perpendicular to the graphene $c$-axis (in-plane), we need $n_{\text {GPerp }}$, while for the electric field parallel to the $c$-axis, we need $n_{\text {GParal }}$. To get these, we use the experimental refractive index taken from the electron energy loss spectroscopy measurements on graphite of ref 37 . For s-polarized light (electric field restricted in the plane), the refractive index to be used is simply $n_{\mathrm{s}}=n_{\text {GPerp }}$. For p-polarization, both inplane and out-of-plane field components exist. Thus, we have an angle-dependent refractive index $n_{\mathrm{p}}^{-2}=n_{\mathrm{GPerp}}^{-2} \cos ^{2} \theta_{i}+$ $n_{\mathrm{GParal}}^{-2} \sin ^{2} \theta_{i}$, where the refracted angle $\theta_{i}$ has to be calculated self-consistently with Snell's law. To account for the numerical aperture in the experiment, we need to integrate the response of all possible incident angles and polarizations with a weight distribution accounting for the Gaussian beam profile used in the experiment $f\left(\theta_{0}\right)=e^{-2 \sin ^{2} \theta_{0} / \sin ^{2} \theta_{\mathrm{m}}} 2 \pi \sin$ $\theta_{0}$, where $\theta_{m}=\arcsin (\mathrm{NA})$.

Figure $4 \mathrm{~b}$ shows the calculated contrast for $N$ between 1 and 6 (lines). This is in excellent agreement with the experiments: (i) the contrast scales with number of layers; (ii) it is maximum at $\sim 570 \mathrm{~nm}$; (iii) no phase shift is observed in this $N$ range. Thus, for $N$ between 1 and 6 , cos $\phi(\lambda=570 \mathrm{~nm})=-1$. The contrast of graphene at $570 \mathrm{~nm}$ is $\sim 0.1$. From eqs 4 and 5 , we get $r_{\mathrm{G}}(\lambda=570 \mathrm{~nm})=0.05$. Thus, $R_{\mathrm{G}}(\lambda=570 \mathrm{~nm})=0.003$. Note that we have an estimated error bar in the contrast measurements of below $5 \%$, with the biggest deviation just for one data point around $560 \mathrm{~nm}$ due to a rapid change of the white light intensity.

It quite remarkable that, without any adjustable parameter, graphene's response can be successfully modeled using 


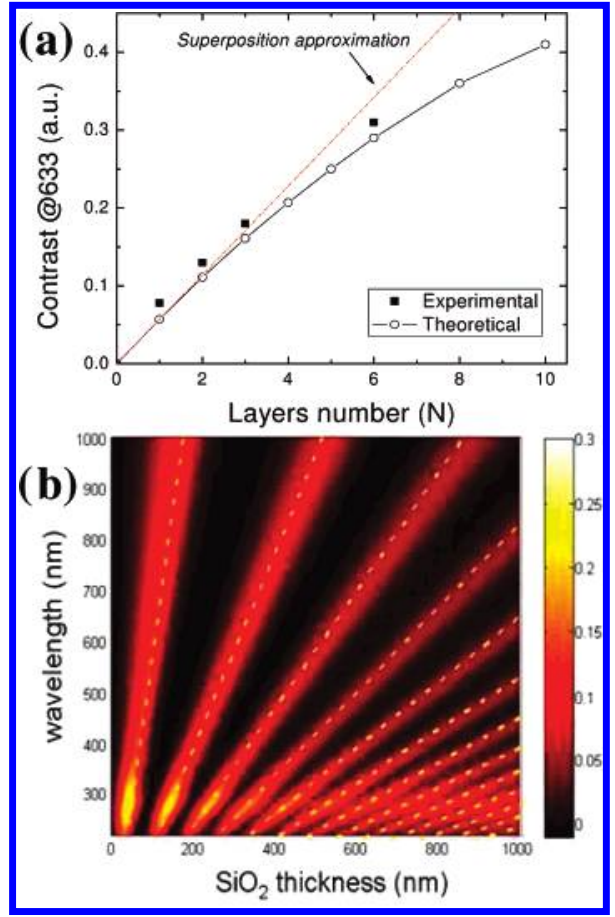

Figure 5. (a) Maximum contrast at $633 \mathrm{~nm}$ as a function of $N$. (b) Calculated contrast of graphene as a function of oxide thickness and excitation wavelength. Dotted lines trace the quarter-wavelength condition.

graphite's dielectric constant. This implies that the optical properties of graphite do not depend on the thickness, i.e., within our experimental uncertainty of $5 \%$, graphene and graphite have the same optical constants. The electrons within each graphene layer form a two-dimensional gas, with little perturbation from the adjacent layers, thus making multilayer graphene optically equivalent to a superposition of almost noninteracting graphene layers. This is intuitive for spolarization. However, quite notably, this still holds when the out-of-plane direction (p-polarization) is considered. This is because, compared to the in-plane case, graphite's outof-plane response is much smaller, and in addition, it gets smeared out by the NA integration. Thus, the maximum contrast $(\lambda=570 \mathrm{~nm})$ of a $N$ layer is: $\delta(N)=0.1 \cdot N$. Figure 6 a shows that this approximation fails for large $N$. When valid, the relation between topography and contrast is given by: $z(\mathrm{~nm})=0.27+3.3 \delta(N)$.

Figure $5 \mathrm{~b}$ plots the contrast as a function of wavelength and $\mathrm{SiO}_{2}$ thickness for a single layer. The maximum contrast occurs at the minima of the background reflectivity. This is expected because this is the most sensitive point in terms of phase matching, and small changes become most visible. Thus, the optimal configuration requires the $\mathrm{SiO}_{2}$ to be tuned as an antireflection (AR) coating, i.e., with its optical length a quarter wavelength. The yellow dotted lines trace the quarter-wave condition $2 n_{\mathrm{SiO}_{2}} d_{\mathrm{SiO}_{2}} / \lambda_{0}=(m+1 / 2)$, and indeed, they closely follow the calculated contrast maxima. This explains why $\sim 300 \mathrm{~nm}$ thick $\mathrm{SiO}_{2}$ allows good contrast in the visible range. However, note that the first maximum is for $\sim 100 \mathrm{~nm}$, which could provide an alternative viable substrate. A second point of interest are the bright spots around $275 \mathrm{~nm}$. These are due to the absorption peak at the

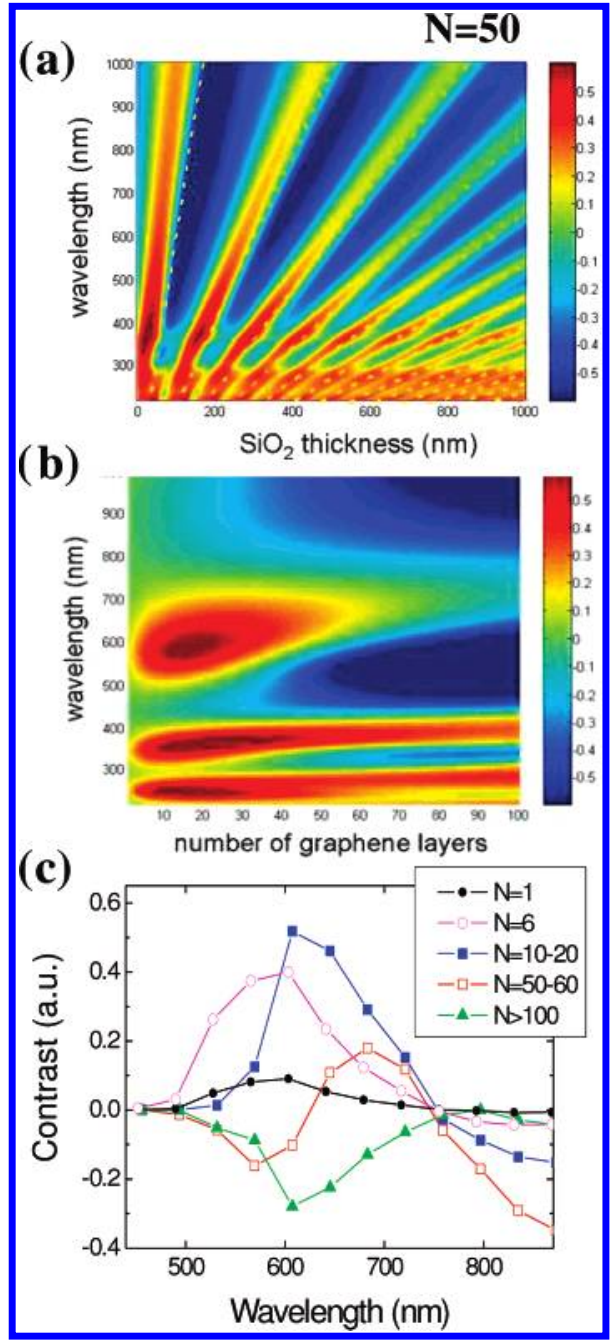

Figure 6. (a) Calculated contrast of 50 layers as a function of oxide thickness and excitation wavelength. (b) Contrast for $300 \mathrm{~nm} \mathrm{SiO}_{2}$ as a function of $N$ and excitation wavelength. (c) Experimental contrast for samples of increasing thickness.

$\pi \rightarrow \pi^{*}$ transition of graphite. ${ }^{37}$ For this excitation, the graphene monolayer not only becomes much more visible, but the contrast change also directly reveals the frequency dependence of the graphene's refractive index. Thus, as for nanotubes, ${ }^{27,28}$ white-light Rayleigh scattering is a direct probe of the dielectric function.

For thicker samples $(N>10)$, the phase change due to the optical path in graphite cannot be neglected. Figure 6a shows the calculated contrast for a 50 layer sample as a function of $\mathrm{SiO}_{2}$ thickness, while Figure $6 \mathrm{~b}$ plots the contrast for a fixed $300 \mathrm{~nm} \mathrm{SiO}{ }_{2}$ thickness but for a variable number of layers. For example, At $600 \mathrm{~nm}$, as $N$ increases, the response first saturates, then decreases and red-shifts, finally becoming negative, as found experimentally (Figure 6c). It is also interesting to note that, for small $N$, the variation along the vertical (wavelength) axis is largely between zero and positive (i.e., reflectivity reduction only), while for a large number of layers, the variation is from positive to negative (i.e., both reflectivity reduction and enhancement). This points to two different mechanisms. For small $N$, the effect of the graphene layers is just to change the reflectivity of the air $-\mathrm{SiO}_{2}$ interface, while they offer no significant optical 


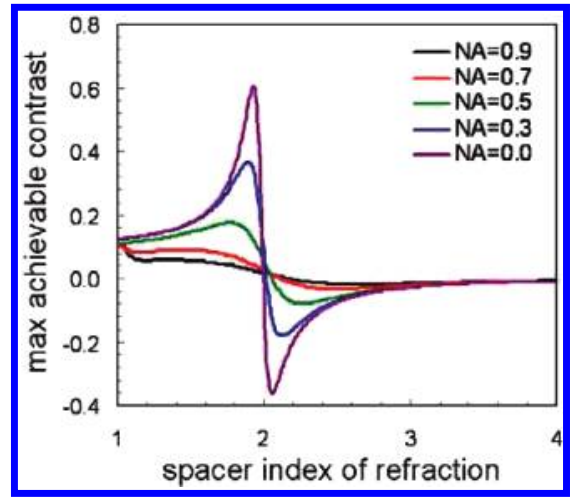

Figure 7. Maximum calculated contrast as a function of spacer refractive index and objective numerical aperture (NA).

depth. For large $N$, on the other hand, the reflectivity of the air-graphene interface saturates while the effect of the increasing optical path within the now thick graphite layer becomes significant. This change is not a monotonic function of $N$. While these two effects are different, they both contribute to a shift of the reflectivity resonance condition, and thus explain the increasing visibility of thicker graphene layers, when measured for a fixed excitation energy.

It is also interesting to consider the contrast as a function of NA. The calculations show that measurements at a reduced NA would give a stronger contrast, as one could intuitively expect. However, there is a nontrivial implication when varying NA if one tries to maximize the contrast by using the antireflection coating rule for the spacer. The ideal AR coating over a substrate of index $n_{\text {subst }}$ must have an index $n_{\text {spacer }}=\sqrt{ } n_{\text {subst }}$ and quarter-wave thickness $d_{\text {spacer }}=(m+$ 1/2) $\lambda_{0} / 2 \sqrt{ } n_{\text {subst. }}$. Because $n_{\mathrm{Si}} \sim 4$ at $600 \mathrm{~nm}$, it is natural to think that a spacer of $n=2$ (e.g., $\mathrm{Si}_{3} \mathrm{~N}_{4}$ ) would be ideal. To explore this, Figure 7 plots the contrast for different NAs as a function of $n_{\text {spacer }}$ at $600 \mathrm{~nm}$ and for spacer thickness $d_{\text {spacer }}$ $=300 \mathrm{~nm}\left(n_{\mathrm{SiO}_{2}} / n_{\text {spacer }}\right)$, which serves to maintain the AR condition and thus the maximum response.

Contrary to expectations, the contrast maximizes for different spacer indexes depending on NA. For normal incidence, it is maximum at 1.93 , with a huge contrast of 0.6 for a single layer, Figure 7. It also has a strong variation thereafter and becomes negative. As NA further increases, the peak moves to a smaller index (around 1.5 for NA = 0.7 ), becomes relatively flat, and eventually goes to $n_{\text {spacer }}$ $=1$. Thus, for large NA, it makes little difference what the spacer index is as long as the quarter-wave condition is satisfied. Indeed, for the ideal AR condition, the background reflectivity goes to zero and thus the contrast becomes large; however, this condition strongly depends on the incidence angle and is thus easily destroyed at large NAs. For all possible spacer refractive indexes, a reduction in NA results into an increased contrast, however, the magnitude of this increase varies: at $n=1.5$ going from 0.7 to 0.0 NA changes the contrast by a factor 2 , while at $n=1.9$, one can gain a factor of 6, Figure 7. For maximum visibility, a $\mathrm{Si}_{3} \mathrm{~N}_{4}$ spacer of thickness 225 or $75 \mathrm{~nm}$ with NA $=0.0$ would be ideal. However, if high resolution is needed, as for nanoribbons or, in general, to analyze edges and defects, a compromise between resolution and image contrast is necessary.

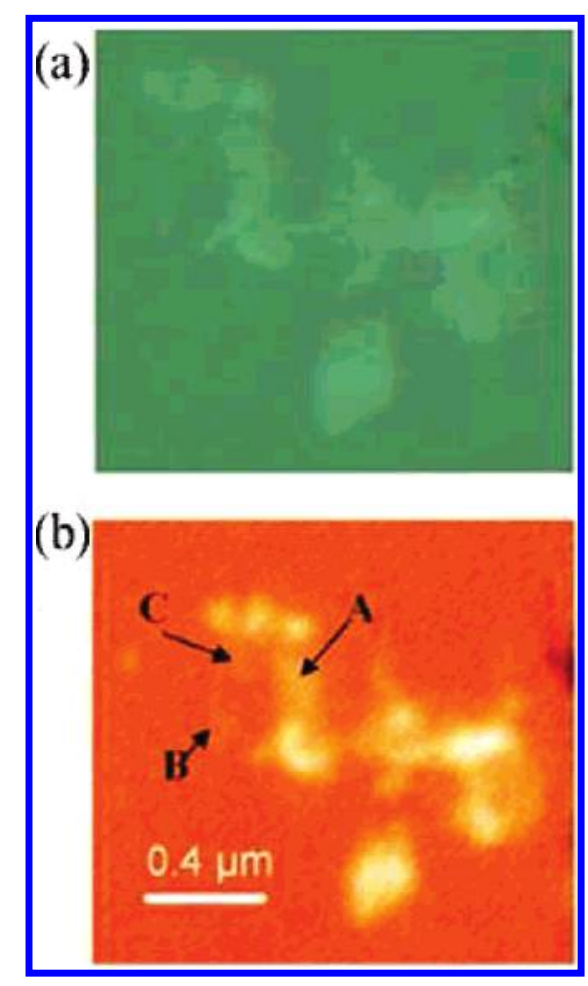

Figure 8. (a) Optical micrograph of flakes on glass. (b) Confocal Rayleigh image at $633 \mathrm{~nm}$ of the same sample area. The flakes marked $\mathrm{A}-\mathrm{C}$ have been characterized by Raman spectroscopy and consist of 7-10 layers (A), 3-6 layers (B), and 1-2 layers (C), respectively. Note the inversion and decrease of contrast compared to the case of the $\mathrm{SiO} 2$ spacer (Figures 3a, 2c, and 4a, respectively).

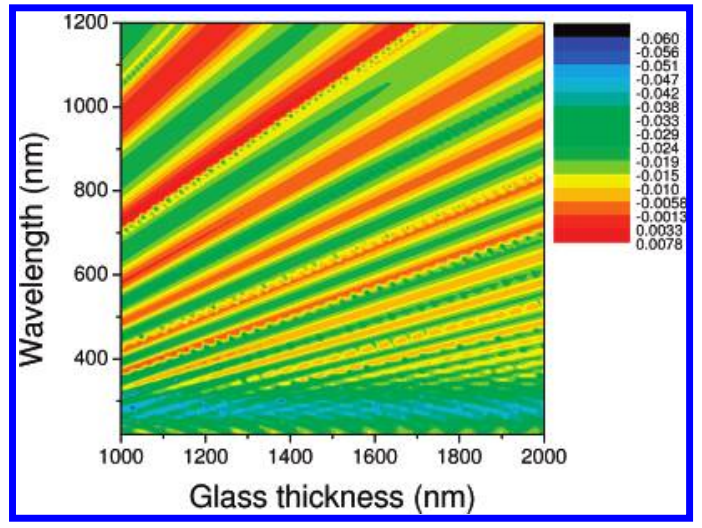

Figure 9. Calculated contrast of graphene on glass at different wavelengths.

A second point to note is that for all NAs the contrast converges to the same value for $n=1$, i.e., for a suspended graphene layer over the substrate. Indeed, optically visible suspended layers were recently reported (see Figure 1 of ref 38). Maximum visibility is achieved if the quarter-wave condition is satisfied, as indeed it is in ref 38 , where the $300 \mathrm{~nm} \mathrm{SiO}_{2}$ spacer is etched to create an air gap between graphene and the $\mathrm{Si}$ substrate. Interestingly, in this case, any measurement with any NA will yield the same contrast. The same considerations are relevant for the case of a thin freestanding spacer (no substrate). By tuning at the low reflection point (now at half-wavelength) and with an NA $=0.0$, one could get fair contrasts. However, as soon as NA increases, 
the resonance condition is destroyed and the contrast becomes much smaller than for the $\mathrm{SiO}_{2} / \mathrm{Si}$ system.

The matrix method can be extended to every film configuration. To prove this, we measure graphene layers on glass. For $N=1$, the calculated contrast at $633 \mathrm{~nm}$ is expected to be $\sim-0.01$. Note the different sign compared with that of the $\mathrm{Si} / \mathrm{SiO}_{2}$ substrate. This is due to the different optical properties of glass and Si. Figure 8a shows an optical micrograph of a multilayer, and Figure $8 \mathrm{~b}$ the corresponding Rayleigh image at $633 \mathrm{~nm}$. Raman spectroscopy shows that the sample is composed of layers of different thickness: A (7-10 layers), B (3-6 layers), C (1-2 layers). The contrast is lower compared to $\mathrm{Si} / \mathrm{SiO}_{2}$, as predicted. Note that the use of UV light could enhance the contrast to $\sim-0.04$ at $300 \mathrm{~nm}$ excitation (Figure $9 \mathrm{~b}$ ).

In conclusion, we used white light illumination combined with interferometric detection to study the contrast between graphene and $\mathrm{Si} / \mathrm{SiO}_{2}$ substrates. We modeled the light modulation by multiple reflections, showing that: (i) the contrast can be tailored by adjusting the $\mathrm{SiO}_{2}$ thickness. Without oxide, no modulation is possible; (ii) the light modulation strongly depends on the graphite thickness. For a few layers $(<6)$, the samples behave as a superposition of single sheets. For thicker samples, both amplitude and phase change with thickness. Thus, Rayleigh spectroscopy provides a simple and quick way to map graphene layers on a substrate. It can also be combined with Raman scattering, which is capable of structural identification.

Acknowledgment. We acknowledge A. K. Geim for useful discussions. C.C. acknowledges support from the Oppenheimer Fund, A.C.F. from the Royal Society and Leverhulme Trust, H.H. from the School of Graduate Studies G. Galilei (University of Pisa), and A.H. from the German Science Foundation (DFG) via the Nanosystems Initiative Munich (NIM).

\section{References}

(1) Geim, A. K.; Novoselov, K. S. Nat. Mater. 2007, 6, 183

(2) Novoselov, K. S.; Jiang, D.; Schedin, F.; Booth, T. J.; Khotkevich, V. V.; Morozov, S. V. Proc. Natl. Acad. Sci. U.S.A. 2005, 102, 10451.

(3) Novoselov, K. S.; Geim, A. K.; Morozov, S. V.; Jiang, D.; Katsnelson, M. I.; Grigorieva, I. V.; Dubonos, S. V.; Firsov, A. A. Nature 2005, 438, 197.

(4) Zhang, Y.; Tan, Y. W.; Stormer, H. L.; Kim, P. Nature 2005, 438, 201.

(5) Novoselov, K. S.; Geim, A. K.; Morozov, S. V.; Jiang, D.; Zhang, Y.; Dubonos, S. V.; Grigorieva, I. V.; Firsov, A. A. Science 2004, 306, 666 .

(6) Novoselov, K. S.; McCann, E.; Morozov, S. V.; Falko, V. I.; Katsnelson, M. I.; Zeitler, U.; Jiang, D.; Schedin, F.; Geim, A. K. Nat. Phvs. 2006, 2, 177

(7) Han, M. Y.; Ozyilmaz, B.; Zhang, Y.; Kim, P. Phys. Rev. Lett. 2007, 98, 206805, cond-mat/0702511.
(8) Lemme, M. C.; Echtermeyer, T. J.; Baus, M.; Kurz, H. IEEE Electron. Device Lett. 2007, 28, 282.

(9) Chen, Z.; Lin, Y. M.; Rooks, M. J.; Avouris, P. 2007, cond-mat/ 0701599.

(10) Zhang, Y.; Small, P. J.; Pontius, W. V.; Kim, P. Appl. Phvs. Lett. 2005, 86, 073104.

(11) Berger, C.; Song, Z.; Li, T.; Li, X.; Ogbazghi, Y.; Feng, R.; Dai, Z.; Marchenkov, A. N.; Conrad, E. H.; First, P. N. J. Phvs. Chem. B 2004, 108, 19912.

(12) Bunch, S. J.; Yaish, Y.; Brink, M.; Bolotin, K.; McEuen, P. L. Nano Lett. 2005, 5, 287.

(13) Viculis, L. M.; Mack, J. J.; Kaner, R. B. Science 2003, 299, 5611.

(14) Viculis, L. M.; Mack, J. J.; Mayer, O. M.; Hahn, H. T.; Kaner, R. B. J. Mater. Chem. 2005, 15, 9.

(15) Niyogi, S.; Bekyarova, E.; Itkis, M.E.; McWilliams, J. L.; Hammon, M. A.; Haddon, R. C. J. Am. Chem. Soc. 2006, 128, 1720.

(16) Stankovich, S.; Dikin, D. A.; Dommett, G. H. B.; Kohlhass, K. M.; Zimmey, E. J.; Stach, E. A.; Piner, R. D.; Nguyen, S. B. T.; Ruoff, R. S. Nature 2006, 442, 282.

(17) Stankovich, S.; Piner, R. D.; Chen, X.; Wu, N.; Nguyen, T.; Ruoff, R. S. J. Mater. Chem. 2006, 16, 155.

(18) Forbeaux, I.; Themlin, J. M.; Debever, J. M. Surf. Sci. 1999, 442, 9.

(19) Ohta, T.; Bostwick, A.; Seyller, T.; Horn, K.; Rotenberg, E. Science 2006, 313, 951.

(20) Rolling, E.; Gweon, G. H.; Zhou, S. Y.; Mun, B. S.; McChesney, J. L.; Hussain, B. S.; Fedorov, A.; First, P. N.; de Heer, W. A.; Lanzara, A. J. Phvs. Chem. Solids 2006, 67, 2172.

(21) Meyer, J. C.; Geim, A. K.; Katsnelson, M. I.; Novoselov, K. S.; Booth, T. J.; Roth, S. Nature 2007, 446, 60.

(22) Ferrari, A. C.; Meyer, J. C.; Scardaci, V.; Casiraghi, C.; Lazzeri, M.; Mauri, F.; Piscanec, S.; Jiang, D.; Novoselov, K. S.; Roth, S.; Geim, A. K. Phvs. Rev. Lett. 2006, 97, 187401

(23) Pisana, S.; Lazzeri, M.; Casiraghi, C.; Novoselov, K.; Geim, A. K.; Ferrari, A. C.; Mauri, F. Nat. Mater. 2007, 6, 198.

(24) Ignatovich, F. V.; Topham, D.; Novotny, L. IEEE J. Sel. Top. Ouantum Electron. 2006, 12, 1292.

(25) Lindfors, K.; Kalkbrenner, T.; Stoller, P.; Sandoghdar, V. Phvs. Rev. Lett. 2004, 93, 0374011.

(26) Failla, A. V.; Qian, H.; Hartschuh, A.; Meixner, A. J. Nano Lett. 2006, 6, 1374

(27) Sfeir, M. Y.; Beetz, T.; Wang, F.; Huang, L. M.; Huang, X. M. H.; Huang, M. Y.; Hone, J.; O’Brien, S.; Misewich, J. A.; Heinz, T. F.; Wu, L. J.; Zhu, M. Y.; Brus, L. E. Science 2006, 312, 554.

(28) Wang, F.; Sfeir, M. Y.; Huang, L.; Huang, X. M. H.; Wu, Y.; Kim, J.; Hone, J.; O’Brien, S.; Brus, L. E.; Heinz, T. F. Phvs. Rev. Lett. 2006, 96, 167401.

(29) Schultz, S.; Smith, D. R.; Mock, J. J.; Schultz, D. A. Proc. Natl. Acad. Sci. U.S.A. 2000, 97, 996

(30) Kim, S. W.; Kim, G. H. Appl. Opt. 1999, 38, 5968.

(31) Calatroni, J.; Guerrero, A. L.; Sainz, C.; Escalona, R. Opt. Laser Technol. 1996, 28, 485.

(32) Born, M.; Wolf, E. Principles of Optics; Pergamon Press: Elmsford, NY, 1959.

(33) Bortchagovsky, E. G.; Fischer, U. C. J. Chem. Phvs. 2002, 117, 5384

(34) Hecht, E. Optics; Addison-Wesley: Reading, MA 1998.

(35) Ciddor, P. E. Am. J. Phvs. 1976, 44, 786.

(36) Palik, E. D., Ed. Handbook of Optical Constants of Solids; Academic Press: New York, 1991

(37) Djurisic, A. B.; Li, E. H. J. Appl. Phvs. 1999, 85, 7404.

(38) Bunch, S. J.; van der Zande, A. M.; Verbridge, S. S.; Frank, I. W.; Tanenbaum, D. M.; Parpia, J. M.; Craighead, H. G.; McEuen, P. L. Science 2007, 315, 490.

NL071168M 